Revista Latinoamericana de la Papa 20 (2): 1 - 13

ISSN: $1853-4961$

http://www.papaslatinas.org/ojs/index.php/index/oai

\title{
Resistencia genética en familias de híbridos de papa (Solanum tuberosum L.) a tizón y estrés hídrico por sequía
}

\author{
I. Zurita ${ }^{1}$, A. Angulo ${ }^{2 / *}$, S. Veramendi ${ }^{2}$, J. La Torre ${ }^{1}$, J. Gabriel ${ }^{2 / 3}$
}

Recibido: $18 / 06 / 2016$

Aceptado: $12 / 09 / 2016$

Accessible en linea: Diciembre 2016

\section{Resumen}

Se evaluaron 265 clones de seis cruzas interespecíficas de Solanum phureja bajo invernadero para resistencia genética a tizón tardío (Phytophthora infestans) y sequía. El diseño experimental fue de filas y columnas y el diseño genético fue el de Carolina del Norte I. Se sometió a ataque de tizón al inicio de tuberización durante dos semanas, tras la recuperación (aplicación de fungicida) se sometió a sequía (suspensión de riego) durante 10 días. Las variables de respuesta fueron: presencia del marcador molecular GP94, ligado al gen de resistencia a tizón $R$-Phi1, Área Bajo la Curva de Progreso de Phytophthora infestans relativa (ABCPPIrel.), grado de marchitez (SEV), recuperación a sequia (REC), número de tallos (NTA), número de hojas $(\mathrm{NH})$, altura de planta (AP), contenido de clorofila (CC), contenido relativo de agua (CRA), peso (PT) y número de tubérculos (NT), Aptitud Combinatoria General (ACG), heredabilidades en sentido amplio $\left(\mathrm{H}^{2}\right)$ y estrecho $\left(\mathrm{h}^{2}\right)$ e importancia de los efectos aditivos (A) y no aditivos (NA). La familia 012-202 fue la más resistente a tizón y sequía. Se encontró correlaciones significativas entre NH, CRA, CC, SEV, REC, ABCPPIrel y PT. Cincuenta y cuatro clones fueron resistentes a tizón y sequía, destacando los clones 012-201-13 y 012-201-45. Los efectos aditivos fueron más importantes en CC, CRA, SEV y ABCPPIrel y NA en REC y NH. La ACG del progenitor CCC81 fue superior para $\mathrm{NH}, \mathrm{CRA}, \mathrm{CC}, \mathrm{SEV}$ y ABCPPIrel. $\mathrm{La} \mathrm{H}^{2}$ fue media- alta para $\mathrm{NH}, \mathrm{CC}$ y ABCPPIrel. La $\mathrm{h}^{2}$ fue alta para CC, CRA y ABCPPIrel. GP94 no detectó la resistencia a tizón.

Palabras clave adicionales: Genes R, severidad, heredabilidad, ACG.

\section{Genetic resistance in families of potato interspecific hybrids (Solanum tuberosum $\mathrm{L}$.) to late blight and drought}

\section{Summary}

Two hundred sixty five clones derived from interspecific crosses of Solanum phureja were evaluated for drought and late blight (Phytophthora infestans) genetic resistance in a greenhouse trial. The experimental design was rows and columns and the genetic design North Carolyn I. The families were under late blight attack at the beginning of tuber initiation during two weeks; after recovery (fungicide application), they were under drought for 10 days (irrigation interruption). The response variables were: presence of molecular marker GP94 (R-Phil gene), relative area under the Phytophthora infestans progress curve (relAUPIPC), wilting severity degree (SEV), drought recovery degree

\footnotetext{
Autor para correspondencia. Correo electrónico: a.angulo@proinpa.org

Universidad Mayor de San Simón. Facultad de Ciencias Agrícolas y Pecuarias "Martín Cárdenas",

Cochabamba, Bolivia.

Fundación PROINPA, Casilla 4285, Cochabamba, Bolivia.

PROMETEO, Universidad Estatal del Sur de Manabí (UNESUM), Jipijapa, Ecuador.
} 
(REC), stem number (SN), leaf number (LN), plant height (PH); chlorophyll content (CC), relative water content (RWC), tuber weight (TW), tuber number (TN), General Combining Ability (GCA), broad and narrow sense heritabilities $\left(\mathrm{H}^{2}\right.$ and $\mathrm{h}^{2}$ respectively) and the importance of additive (A) and non-additive (NA) genetic effects importance. The family 012-202 was the most resistant to drought and late blight. Significant correlations among LN, RWC, CC, SEV, REC, relAUPIPC and TW were found. 012-201-13 and 012-201-45 were outstanding among 54 clones found resistant to drought and late blight. The additive effects were more important to CC, RWC, SEV and relAUPIPC. NA was more important to REC and LN. CCC81 parent had the highest GCA for LN, RWC, CC, SEV and relAUPIPC. LN, CC and relAUPIPC have high $\mathrm{H}^{2}$ and CC, RWC and relAUPIPC high $\mathrm{h}^{2}$. GP94 did not identify late blight resistance.

Additional Key words: R genes, severity, heritability, GCA.

\section{Introducción}

La papa (Solanum tuberosum L.) es el cuarto cultivo más importante a nivel mundial, siendo superado solamente por la caña de azúcar, el maíz y el trigo; y situándose por encima de cultivos como la remolacha azucarera, la mandioca y la soya (FAO, 2012). En Bolivia es el tubérculo más cultivado con cerca de 130 mil hectáreas (INE, 2011), e involucra aproximadamente a 203 mil familias de agricultores (Zeballos et al., 2009). El rendimiento promedio es de 5,14 t/ha, siendo el más bajo en Sud América (FAO, 2012), respecto de países como: Argentina, Brasil y Chile, donde alcanzan rendimientos de 31,43, 26,81 y 26,33 t/ha respectivamente (FAO, 2012). A nivel mundial países como Nueva Zelanda, Bélgica y Países Bajos alcanzan rendimientos de 47,5, 45,42 y 45,17 t/ha respectivamente (FAO, 2012). Los bajos rendimientos se atribuyen a diversos factores abióticos como ser las heladas y las sequías y a factores bióticos como el ataque de plagas y enfermedades. Entre las enfermedades el tizón causado por el oomycete Phytophthora infestans (Gabriel, 2010), es uno de los problemas más importantes en el cultivo de la papa a nivel mundial y nacional. En Bolivia se estima que 40 mil familias de pequeños agricultores son afectados por esta enfermedad, perdiendo entre 25 a 30 millones de dólares anualmente (Fernández-Northcote et al., 2000), con reducciones en el rendimiento de hasta el $100 \%$. Para el control del tizón se recurre al control cultural (densidad que favorezca la aireación, manejo de semilla certificada, riego controlado, etc.), control químico (aplicación oportuna de fungicidas) y la utilización de cultivares resistentes y/o tolerantes. La sequía es otro de los principales problemas para la baja producción, así en el año 2010 se reportó como 80 mil familias afectadas por sequía, el doble con respecto al año 2007 (INE, 2011), registrándose pérdidas en rendimiento de más del $50 \%$. Para hacer frente a este problema, se puede recurrir a alternativas como: la optimización del agua, o la obtención de cultivares tolerantes y/o resistentes. La primera opción aplicaría para cultivos bajo riego, en tanto que la segunda opción aplicaría a cultivos a secano principalmente (Larcher, 1995). Los marcadores moleculares permiten la selección asistida reduciendo el periodo de evaluación y selección para caracteres como tamaño de tubérculo, resistencia a tizón tardío y otras enfermedades (Bradshaw et al., 2008). Por los antecedentes mencionados el presente trabajo tuvo los objetivos de: i) obtener clones de papa resistentes al tizón tardío, ii) obtener clones resistentes al estrés hídrico por sequía, iii) determinar los mecanismos asociados con la resistencia a sequía y a tizón, iv) establecer las bases genéticas de la resistencia al tizón tardío 
y al estrés hídrico por sequía y v) determinar aquellos clones que poseen genes $\mathrm{R}$ favorables de resistencia a tizón mediante la aplicación del marcador molecular GP94.

\section{Materiales y métodos}

El ensayo se realizó en un invernadero de la Fundación PROINPA a $15 \mathrm{~km}$ de la ciudad de Cochabamba, entre los paralelos $17^{\circ} 18^{\prime}$ de latitud Sud, y $66^{\circ}$ $14^{\prime}$ de longitud Oeste y una altitud de $2540 \mathrm{msnm}$. Se utilizó semilla sexual de seis familias de papa (70 semillas/familia) provenientes de cruzamientos entre cultivares de las especies Solanum andigena (4x), $S$. tuberosum (4x), $S$. phureja (2x), $S$. goniocalyx (2x) y S. stoloniferum $(4 x)$ (Tabla 1). La semilla se trató con ácido giberélico a 2500 ppm durante 24 horas. Una vez que las plántulas tuvieron dos hojas verdaderas, se trasplantaron a macetas de un kilogramo. De los 420 clones sembrados, sobrevivieron 265 , los cuales se evaluaron para los estudios de resistencia.

\section{Metodología para resistencia a tizón}

Presencia del gen de resistencia Rpiphul. Antes de la inoculación y en estado de plántula se evaluó la presencia del gen/QTL Rpi-phulde resistencia para tizón, ligado y co-localizado con el marcador CAP, GP94 (Ritter et al., 2005; Ritter et al., 2008).

Extracción de ADN. Se tomó dos foliolos de cada planta, cuando las plantas tenían tres hojas verdaderas y se conservaron a $20{ }^{\circ} \mathrm{C}$. Las muestras fueron molidas en nitrógeno líquido a $-195^{\circ} \mathrm{C}$. Se utilizaron $100 \mathrm{mg}$ para el proceso de extracción de ADN genómico mediante el protocolo de CTAB 2X (hexadecil bromuro de trimetil amonio) según Doyle y Doyle (1990).

Cuantificación de ácidos nucleicos. Se realizó mediante electroforesis en geles de agarosa al $1 \%$. Las muestras migraron por 45 minutos a una potencia de $70 \mathrm{~V}$ $(5 \mathrm{v} / \mathrm{Cm})$ y se visualizaron a través de un transluminador UV marca Biorad. La calidad y concentración del ADN genómico se valoró por comparación de intensidad con las bandas del marcador de peso molecular de concentración conocida (10.000 bp Eurogentec).

PCR y digestión. Un volumen de $15 \mu \mathrm{L}$ de mezcla (4 ng de DNA Molde, $1 \mathrm{X}$ de Tampón PCR 10X, 0,2 mM dNTP, 1 $\mathrm{pmol} / \mu \mathrm{L}$ de cada iniciador y $0,025 \mathrm{U} / \mu \mathrm{L}$ de la enzima Taq polimerasa) se amplificaron en un termociclador (modelo PTC-100, MJ Research, Ramsey, Minnesota, USA), bajo un tiempo de desnaturalización inicial de cinco minutos a $94^{\circ} \mathrm{C}$, desnaturalización a $94^{\circ} \mathrm{C}$ por un minuto, 35 ciclos de anillamiento a $94^{\circ} \mathrm{C}$ por un minuto, $\mathrm{X}^{\circ} \mathrm{C}$ por $45 \mathrm{~s}$ y $72^{\circ} \mathrm{C}$ por 5 minutos. Los productos de amplificación fueron visualizados y cuantificados mediante electroforesis en geles de agarosa al $1,8 \%$, con $5 \mu \mathrm{L}$ del producto PCR y 0,5 $\mu \mathrm{L}$ de tampón de carga y una corriente de $5 \mathrm{~V} / \mathrm{cm}$. Posteriormente se digirieron utilizando $20 \mu \mathrm{L}$ de mezcla $(5 \mathrm{ng} / \mu \mathrm{L}$ de DNA Molde, Buffer ER 10X, BSA 10 $\mu \mathrm{g} / \mu \mathrm{L}$ y $10 \mathrm{u} / \mu \mathrm{L}$ de la enzima $D d e 1)$. Los productos digeridos se separaron por electroforesis en gel de agarosa al 2\% (2 $\mathrm{g}$ de agarosa y $100 \mathrm{~mL}$ de TBE $1 \mathrm{X}$ ). La muestra se preparó con $10 \mu \mathrm{L}$ del producto digerido y $2 \mu \mathrm{L}$ de tampón de carga. Los geles se visualizaron en el transluminador marca Biorad.

Grado de severidad o resistencia a tizón (ABCPPIrel). La infestación natural de tizón fue con una raza compleja de la zona, la misma se presentó a los 60 días después de la siembra, que coincidió con el inicio de la tuberización en la mayoría de los genotipos. Se hizo las lecturas de severidad cada tres días, realizándose un total de cuatro lecturas con la escala sugerida por Henfling (1982). Posteriormente se estimó el área relativa bajo la curva de progreso de P. infestans (ABCPPIrel.) (CIP, 2009). Una vez concluidas las lecturas de severidad, se 
aplicó un fungicida para el control de la enfermedad y promover la recuperación de las plantas.

Metodología para la evaluación de la resistencia a sequía. Una semana después de finalizada la evaluación de tizón, se inició el tratamiento de sequía. Se aplicó un riego controlado de $500 \mathrm{~mL}$ de agua, para posteriormente suspender el riego durante 10 días tal como recomiendan Gabriel et al. (2011a). Al cabo de los 10 días se evaluaron las siguientes variables:

Grado de Marchitez por estrés hídrico por sequía, grado de severidad o resistencia a sequia (SEV). Se evaluó con la escala de Blum (1993) adaptada por Angulo et al. (2005).

Número de tallos (NT), número de hojas (NH) y altura de planta (AP).

Contenido de clorofila (CC). Se evaluó con el SPAD 520, se tomaron cinco lecturas de una hoja tomada al azar del tercio superior de la planta. Se promedió estos valores.

Contenido relativo de agua (CRA). En una hoja, se registró el peso fresco (PF), luego se sumergió en agua destilada por seis horas y se registró el peso turgente (PT), después se secó a $70^{\circ} \mathrm{C}$ hasta llegar a un peso constante y se registró el peso seco (PS), finalmente se calculó el porcentaje de CRA con la siguiente fórmula: CRA $(\%)=(\mathrm{PF}-\mathrm{PS}) /(\mathrm{PT}-\mathrm{PS})$.

Recuperación después del Estrés (REC). Una vez evaluadas las variables SEV, $\mathrm{NH}$, NT, AP, CC y CRA, se regó las plantas con $500 \mathrm{~mL}$, y pasados cinco días se evaluó el REC, según la escala de 1 a 5 de Blum (1993) modificada para REC donde 5 era una planta completamente recuperada y 0 una planta muerta.

Rendimiento. A la cosecha se evaluó el peso (PT) y número de tubérculos (NTU).

\section{Análisis estadísticos}

Se utilizó el diseño de Filas y Columnas para controlar la variación de la luz en el invernadero. El número de clones/familia fueron las repeticiones. Se realizó el análisis de varianza (ANVA) para SEV, REC y ABCPPIrel y el análisis de correlación de Pearson entre todas las variables evaluadas para tizón y sequía, utilizando el programa estadístico SAS V.8 (1999). Para discriminar los genotipos por su resistencia a tizón y sequía se realizó el análisis de agrupamiento (Cluster) de acuerdo al Método de Ward, utilizando el Análisis cluster jerárquico del SPSS.

\section{Análisis genético}

Para el análisis genético se tomó a los progenitores de cada familia como factores. Para el cálculo de la Aptitud Combinatória General (ACG) los factores fueron de interés particular (fijos). Para el cálculo de heredabilidades, fueron de interés poblacional (aleatorios). Las cruzas se realizaron bajo el diseño I de Carolina del Norte (Márquez y Sahagún, 2012). Este diseño permite el cálculo de parámetros de varianza solo para el progenitor masculino; siendo imposible un cálculo de parámetros (ACG) para progenitores femeninos y de interacción (Aptitud Combinatoria Especifica o ACE). Si bien este diseño no permite un análisis completo, el bajo costo y la facilidad del mismo implican un trabajo más simple (Gardner, 1963). Las varianzas y heredabilidades se calcularon utilizando el programa estadístico SAS V.8 (1999).

\section{Resultados y discusión}

\section{Resistencia en familias}

Resistencia a Tizón Tardío. El ANVA mostró diferencias significativas para el ABCPPIrel entre familias $(\mathrm{p}<0,01)$; la comparación de medias (Figura 1) determinó que 012-202 es más resistente a tizón $(0,2465)$ que $012-205(0,4946)$ y 
012-206 (0,6576); en cambio, 012-201 $(0,3292), \quad 012-200 \quad(0,3527)$ y $012-207$ $(0,4604)$ presentan una resistencia igual a las susceptibles 012-205 y 012-206 (Figura 1). Gabriel et al. (2007) reportaron resistencia parcial al tizón conferida por DB226 (S. phureja). La resistencia al tizón se conoce es poligénica (Wastie, 1991), encontrándose ocasionalmente genotipos susceptibles en familias resistentes (Gabriel et al., 2011b). En la familia resistente 012-202 se encontró un $30 \%$ de genotipos susceptibles. Por otra parte, en la familia 012-207 que proviene de padres resistentes fue casi completamente susceptible.

Resistencia a Sequia. El ANVA para SEV, mostró diferencias significativas entre familias $(\mathrm{p}<0,01)$; la comparación de medias (Figura 2) mostró que la familia 012-202 fue más resistente a sequía $(2,92)$ que las demás $(3,53-3,90)$. Gabriel et al. (2013) encontraron que Salomé (progenitor de 012-202) presentó resistencia moderada a sequía; frente a otras susceptibles como Desireé. Por otra parte, el ANVA para REC señalo diferencias significativas entre familias $(\mathrm{p}<0,01) ; 012-202$ recuperó mejor $(4,49)$ que 012-205 (2,50); sin embargo, fue estadísticamente igual a 012-200, 012$201,012-206$ y 012-207 (3,43-3,09), que a su vez son iguales a 012-205 (Figura 3). El alto REC de 012-202 posiblemente fue heredado de Salome, que proviene de India [tbr x (tbr x sto)]. Gabriel et al. (2011a) encontraron que India expreso un alto REC.

\section{Análisis de correlación}

La SEV se asoció negativamente con $\mathrm{NH}$ en 012-205; con CRA en 012-201, 012205 y 012-206; con REC en 012-201, 012-205, 012-206 y 012-207; y con ABCPPIrel en 012-201 y 012-202 (Tabla 2). Los clones resistentes a sequia presentaron mayor NH, CRA y REC en 012-205, mayor CRA, resistencia a tizón y REC en 012-201, mayor CRA y REC en 012-206, mayor resistencia a tizón en 012-202 y mayor REC en 012-207 (Tabla 2). Ancasi (2014) encontró también una correlación negativa entre SEV y REC en familias de híbridos de papa. Gabriel et. al. (2011a); en un ensayo de resistencia a sequía que incluía a Puyjuni imilla (progenitor de 012-201) y a India (ancestro de 012-202); evidenciaron una alta y negativa correlación entre SEV y REC. Por otra parte, Deblonde y Ledent (2001); en papa sometida a sequía también encontraron que el NH disminuía con la SEV. Gabriel et. al. (2015) encontraron; asimismo, una relación alta y negativa entre CRA y SEV en variedades nativas de papa bajo sequía.

El REC se asocia positivamente a $\mathrm{NH}$ en 012-201 y 012-205; a CRA en 012-206; a CC en 012-201; y a PT en 012-206. Esto significa que los clones que mejor recuperaron presentaron mayor $\mathrm{NH}$ en 021-205, mayor CRA y PT en 012-206 y mayor NH y CC en 012-201 (Tabla 2). Anithakumari et al. (2012), en una población proveniente de $S$. phureja y $S$. tuberosum sometida a sequia; encontraron una asociación negativa entre SEV y CC. Este resultado corrobora la asociación positiva entre REC y CC en el presente estudio.

El ABCPPIrel se asoció negativamente con el CRA en 012-206 y con el CC en 012-202. Los clones más resistentes a tizón presentaron mayor CRA en 012-206 y mayor CC en 012-202 (Tabla 2).

\section{Análisis Clúster}

El Análisis Clúster se realizó para todos los clones que produjeron tubérculos (218). El dendograma diferenció cinco grupos. El grupo tres (Figura 4) mostró 54 clones (Tabla 3) con las menores SEV y ABCPPIrel. y las mayores REC, NH, CRA, NT y PT . Destacan los clones 012201-13 y 012-201-45 (Tabla 3).

\section{Análisis genético}

Aptitud Combinatoria General (ACG). Existen diferencias significativas 
$(\mathrm{p}<0,01)$ entre las ACGs de los progenitores DB-22670 y CCC81 para NH, CRA, CC, SEV y ABCPPIrel (Tabla 4).

ACG para resistencia a tizón. CCC81 expresó la mejor ACG ya que disminuyó en 11,40 el daño por ataque de tizón (Tabla 4). Asimismo, Gabriel et al. (2007) encontraron que CCC81 fue más resistente a tizón que Robusta y Desirée. Por su parte, Visker et al. (2004), observaron que CE51 (descendiente de $S$. phureja) presentó la mayor ACG para resistencia a tizón.

ACG para resistencia a sequía. CCC81 disminuyó en 0,20 el SEV en sus progenies (Tabla 4). Gabriel et al. (2012) encontraron que YHxblb21 progenie de la cruza entre CCC81 con $S$. bulbocastanum, expresó el mejor aporte a resistencia a sequía (-1.10 SEV) y $(0,59$ REC). CCC81 también expresó las ACGs más propicias para $\mathrm{NH}, \mathrm{CRA}$ y $\mathrm{CC}$ (Tabla 4). Se observó un incremento de 1,18 hojas, 5,56 en CRA y 3,55 en CC.

\section{Heredabilidades}

Resistencia a tizón. El ABCPPIrel expresa heredabilidades en sentido estrecho $\left(\mathrm{h}^{2}\right)$ y amplio $\left(\mathrm{H}^{2}\right)$ de 0,71 (Tabla 5). El $71 \%$ de lo observado se debe al genotipo y se transmite a las progenies. Por tanto, para esta variable solo existen efectos aditivos. Asimismo, Trognitz et al. (2001), en un ensayo de resistencia a tizón tardío en una progenie diploide de papa, encontró que el porcentaje promedio de área foliar enferma (asociada altamente con el ABCPPI) presenta una $\mathrm{H}^{2}$ moderada alta. Del mismo modo, Christ y Haynes (2001) encontraron que la resistencia a tizón tardío en papa diploide presenta efectos aditivos predominantes.

Resistencia a sequía. Los valores de $\mathrm{H}^{2}$ y $\mathrm{h}^{2}$ para SEV y REC fueron de 0,38 y 0,40 y de 0,23 y 0,10 respectivamente (Tabla 5). En SEV el $38 \%$ de lo observado se debe al genotipo y el $23 \%$ puede heredarse a la progenie; en REC el $40 \%$ de lo observado se debe al genotipo y solo el $10 \%$ se transmite a las progenies. En SEV los efectos aditivos son más importantes que los no aditivos $(1,62)$; contrariamente en REC los no aditivos son más importantes $(3,12)$. En contraposición, Ancasi (2014) en un ensayo de sequía en papa bajo invernadero encontró $\mathrm{H}^{2}$ altas para SEV y REC $(0,87$ y 0,79 respectivamente); asimismo, encontró que SEV y REC presentaron efectos aditivos equivalentes a los no aditivos. Por otra parte, $\mathrm{NH}$, CRA y CC tuvieron valores de $\mathrm{H}^{2}$ de $0,75,0,46$ y 0,66 respectivamente; en tanto que las $\mathrm{h}^{2}$ correspondientes fueron de $0,07,0,46$ y 0,66 . Para $\mathrm{NH}$ los efectos no aditivos fueron más importantes $(9,98)$; en tanto para CRA y CC no hubo efectos no aditivos apreciables (Tabla 5).

\section{Marcadores Moleculares}

Marcador GP 94. El marcador GP-94 se observó en todos los genotipos, excepto en 012-205-42 y 012-205-43; sin embargo, hubo variabilidad en la respuesta fenotípica de la resistencia a tizón (resistencia poligénica). Juyó et al. (2010) también encontraron susceptibilidad a tizón asociada a la presencia del alelo de resistencia R11800 en cultivares de $S$. tuberosum subsp. tuberosum. Esto contrasta con Sliwka et al. (2010), que en un ensayo de selección de variedades de papa con marcadores moleculares corroboró los resultados obtenidos en campo. Según Mullins et al. (2006), la papa presenta rasgos poligénicos altamente influenciados por ambiente. Por tanto, la presencia del marcador podría no expresar resistencia a tizón.

\section{Conflictos de Intereses}

Esta investigación fue desarrollada por la Fundación PROINPA y no presenta conflictos de interés. 


\section{Agradecimientos}

Este trabajo fue desarrollado por la Fundación PROINPA, en el sub-proyecto "Desarrollo de variedades con tolerancia a factores abióticos" ejecutado en el marco del Programa Nacional de Papa del INIAF. Se realizó con ayuda financiera del Banco Mundial. Las opiniones expresadas en el mismo no reflejan necesariamente la opinión oficial de estas instituciones.

\section{Referencias citadas}

Acuña, I. 2008. (INIA-Chile) Manejo integrado del tizón tardío y estrategias de control químico. pp 2.

Ancasi, R. 2014. Resistencia genética de híbridos inter-específicos de papa (Solanum tuberosum L.) Al estrés hídrico por sequía en invernadero. Tesis papa obtener el grado de Licenciatura. Universidad Mayor de San Simón, Facultad de Ciencias Agrícolas Pecuarias Forestales y Veterinaria "Dr. Martín cárdenas". pp 22-34.

Angulo, A.; Siles, M.; Ríos, R. y Gabriel, J. 2005. Caracterización de 118 accesiones de arveja (Pisum sativum L.) del Banco de Germoplasma del Centro de Investigaciones Fitoecogenéticas de Pirumani para resistencia a sequía. Revista de Agricultura 42 (60): 25-31.

Anithakumari, A. 2012.Genetic dissection of drought tolerance and recovery potential by quantitative trait locus mapping of a diploid potato population. Mol. Breeding (2012) 30 (3):1413-1429.

Blum, A. 1993. Selection for sustained production in water deficit environments, Crop Sci. I: 343-347.

Christ, B. y Haynes, K. 2001. Inheritance of resistance to early blight disease in a diploid potato population Plant Breeding 120:169-172

CIP (Centro Internacional de la Papa) 2009. Procedimientos para pruebas de evaluación estándar de clones avanzados de papa. pp 53.
Deblonde, P. y Ledent, J. 2001. Effects of moderate drought conditions on green leaf number, stem height, leaf length and tuber yield of potato cultivars. Eur. J Agro 14:31-41.

Doyle, J. J. y Doyle, J. L.1990. Isolation of DNA from small amounts of plant tissues.BRL Focus 12: 13-15

FAO (Food and Agriculture Organization) 2012. Disponible en: http://faostat.fao.org/site/567/DesktopDefa ult.aspx?PageID=567\#ancor (Consulta: Agosto 2013).

Fernández - Northcote, E.; Navia, O. y Gandarillas, A. 2000. "Basis of strategies for chemical control of potato late blight developed by PROINPA in Bolivia". Fitopatología 35 (3): 137-149

Gabriel, J.; Forqueda, F.; Plata, G. y Fernández, E. 2007. Caracterización de genotipos de papa de Europa y Latinoamérica por resistencia a tizón y propiedades culinarias. Revista Latinoamericana de la Papa. 14 (1): 10-23.

Gabriel, J. 2010. Documento marco: Estrategias y perspectivas del mejoramiento genético de papa (Solanumtuberosum L.) en Bolivia. Fundación PROINPA, Cochabamba, Bolivia. pp 60.

Gabriel, J.; Porco, P.; Angulo, A.; Magne, J.; La Torre, J. y Mamani, P. 2011a. Resistencia genética a estrés hídrico por sequia en variedades de papa (Solanum tuberosum L.) bajo invernadero. Revista Latinoamericana de la Papa 16 (2):173208.

Gabriel, J.; Orellana, L.; Plata, G. y Siles, M. 2011b. Aptitud combinatoria de la resistencia al tizón tardío (Phytophthora infestans) en cultivares nativos de papa Revista Latinoamericana de la Papa 16 (1): 85-98.

Gabriel, J.; Ancasi, G.; Angulo, A.; Magne, J.; La Torre, J. y Mamani, P. 2012. Genetically-induced drought resistance in potato hybrids (Solanum tuberosumL.). Revista Latinoamericana de la Papa 17 (1):97-124 
Gabriel, J.;Veramendi, S.; Angulo, A. y Magne, J. 2013. Respuesta de variedades mejoradas de papa (Solanum tuberosumL.) al estrés hídrico por sequía. J Selva Andina Biosph. 1 (1):25-36.

Gabriel, J.; Canqui, J.; Cadima, X. y Angulo, A. 2015. Respuesta de ocho cultivares nativos de papa (Solanum tuberosum L.) al estrés hídrico por sequía en la comunidad de Larati, Sacaba (Cochabamba). Cochabamba, Bolivia. pp 15 (Artículo en preparación).

Henfling, J. 1982. Field screening procedures to evaluate resistance to late blight.International Potato Center.Technology evaluation series No. 1982-5.pp 15.

INE Instituto Nacional de Estadística de Bolívia 2011. Bolívia: Eventos adversos de origen natural reportados, famílias damnificadas por eventos adversos de origen natural, según tipo de evento, 20032011.Disponible en: http://www.ine.gob.bo/indice/general.aspx? codigo $=40104 \mathrm{http}: / / \mathrm{www}$.ine.gov.bo/indice /EstadísticaSocial.aspx?codigo=80601.

(Consulta: Agosto 2013).

Juyó, D.; Gerena, H. y Mosquera, T. 2010. Evaluación de marcadores moleculares asociadoscon resistencia a gota (Phytophthora infestansL.) en papas diploides y tetraploides. Rev. Colomb. Biotecnol. 13 (2):51-62.

Larcher, W. 1995.Physiological plant ecology.Ecophysiology and stress physiology of functional groups. SpringerVerlag, Berlin-Heidelberg. pp 401-416.

Márquez, F. y Sahagún, J. 2012. Estimación de varianzas genéticas en maíz con familias independientes de hermanos completos. Universidad Autónoma Chapingo, México. Agrociencia 34: 437444.

Mullins, E.; Milbourne, D.; Petti, C.; Doyle-Prestwich, B. y Meade, C. 2006.Potato in the age of biotechnology. Trends in Plant Sci 11: 254-260.

Ritter, E.; Lucca, F.; Sánchez, I. y Ruiz de Galarreta, J. I. 2005. Genomic resources in potato and possibilities for exploitation. In: Haverkort AJ, Struik PC et al. (eds) Potato in progress; science meets practice. Wageningen Academic Publishers, the Netherlands, pp 55-65

Ritter, E.; Ruiz de Galarreta, J. I.; Van Eck, H. J. y Sánchez, I. 2008. Construction of a potato transcriptome map based on thec DNA-AFLP technique. Theor. Appl. Genet. 116:1003-1013

SAS 1999.The SAS System for windows V8.SAS Institute Inc., Cary, N.C. USA

Sliwka, J.; Jakuczun, H.; Kamiñski, P. y Zimnoch, E.2010. Marker-assisted selection of diploid and tetraploid potatoes carrying Rpi-phu1, a major gene for resistance to Phytophthora infestans. J. Appl. Genet. 51 (2):133-140.

Trognitz, B.; Orrillo, M.; Portal, L.; Román, C.; Ramón, P.; Perez, S. y Chacón, G. 2001.Evaluation and analysis of reduction of late blight disease in a diploid potato progeny. DOI: $10.1046 / j .1365-$ 3059.2001.00567.x. Disponible en: http://onlinelibrary.wiley.com/doi/10.1046/ j.1365-3059.2001.00567.x/full (Consulta: Septiembre 2014)

Visker, M.; Van Raaij, H.; Keizer1, L.; Struik, P. y Colon, L. 2004. Correlation between late blight resistance and foliage maturity type in potato. Euphytica 137: 311-323.

Wastie, R. L. 1991. Breeding for resistance. Adv. Plant. Pathol. 7:193-223.

Zeballos, H.; Balderrama. F.; Condori, B. y Blajos, J. 2009.Economía de la papa en Bolivia (1998-2007). Fundación PROINPA, Cochabamba, Bolivia. pp 129. 
Tabla 1.Genealogía de seis familias de papa de cruzamientos inter específicos.

\begin{tabular}{llllll}
\hline \multirow{2}{*}{$N^{o}$} & \multirow{2}{*}{ Codificación } & \multirow{2}{*}{ Familia } & \multicolumn{2}{c}{ Genealogía } & \\
& & & Madre & Padre & Clones \\
\hline 1 & f0 & $012-200$ & CCC81(r) & CCC81(r) & 30 \\
2 & f1 & $012-201$ & Puyjuni (r) & CCC81 & 62 \\
3 & f2 & $012-202$ & Salomé (r) & CCC81 & 36 \\
4 & f5 & $012-205$ & Chulina 3(s) & DB-22670(r) & 60 \\
5 & f6 & $012-206$ & Candelaria 1(r) & DB-22670 & 51 \\
6 & f7 & $012-207$ & Chulina 1(r) & DB-22670 & 26 \\
\hline
\end{tabular}

CCC81 = phu, Puyjuni $=$ India $\times$ Waych'a, Salomé $=$ India $\times$ phu + gon, Chulina $1=$ phu, Chulina 3 = phu, Candelaria 1= phu, DB-22670= phu, India = tbr x (tbr x sto), Waych'a $=\operatorname{adg}$, phu $=S$. phureja, gon $=$ S. goniocalyx, tbr $=S$. tuberosum, sto $=S$. stoloniferum, adg $=S$. andigena, $(\mathrm{r})=$ resistente a tizón, $(\mathrm{s})=$ susceptible a tizón. Fuente: López et al., (2011), Gabriel et al. (2011a).

Tabla 2. Correlaciones significativas entre el ABCPPIrel, cinco variables relacionadas con resistencia a sequía y una variable de rendimiento en cinco familias de papa.

\begin{tabular}{|c|c|c|c|c|c|c|c|c|}
\hline Familia & & NH & CRA & $\mathbf{C C}$ & SEV & REC & ABCPPIrel & PT \\
\hline & & $\left(\mathbf{n}^{\circ}\right)$ & $(\%)$ & & & & & $(\mathbf{K g})$ \\
\hline \multirow[t]{6}{*}{ 012-201 } & SEV & $-0,30$ & $-0,46$. & $-0,24$ & 1,00 & $-0,73$ & 0,60 & $--0,01$ \\
\hline & & 0,02 & $-\leq 0,01$ & $-0,08$ & & $\leq \mathbf{0 , 0 1}$ & $\leq \mathbf{0 , 0 1}$ & 0,94 \\
\hline & REC & $-0,46$ & $--0,26$ & 0,60 & $-0,73$ & $-1,00$ & $-0,42$ & $-0,25$ \\
\hline & & $<0,01$ & 0,05 & $\leq 0,01$ & $<0,01$ & & $\leq 0,01$ & 0,06 \\
\hline & AВСРPIrel & $-0,16$ & $-0,38$ & 0,06 & $\mathbf{0}, \overline{60}$ & $-0,42$ & 1,00 & $<0,01$ \\
\hline & & 0,23 & $<0,01$ & 0,67 & $<0,01$ & $<0,01$ & & 0,10 \\
\hline \multirow[t]{4}{*}{ 012-202 } & SEV & $---0,03$ & $--0,41$ & $-0,45$ & 1,00 & $-0,34$ & 0,59 & 0,31 \\
\hline & & -ーーー- 0,86 & $-0,01$ & -0,01 & & $-0,04$ & $\leq \mathbf{0 , 0 1}$ & 0,07 \\
\hline & AВСPPIrel & $---0,15$ & $-0,42$ & 0,52 & 0,59 & $-0,15$ & -1,00 & 0,21 \\
\hline & & 0,37 & $0, \overline{01}$ & $<0,01$ & $<0, \overline{01}$ & 0,38 & & 0,21 \\
\hline \multirow[t]{4}{*}{ 012-205 } & SEV & $----\underline{-0,60}$ & $-0,47$ & $-0,09$ & 1,00 & $-\underline{-0,74}$ & $-0,14$ & $-0,04$ \\
\hline & & $-ー-ー \leq \mathbf{0}, \mathbf{0 1}$ & $\leq \mathbf{0 , 0 1}$ & $-0,60$ & & $\leq \mathbf{0 , 0 1}$ & $-0,38$ & $0, \underline{0} 0$ \\
\hline & REC & $0,0,57$ & 0,34 & $-0,40$ & $-0,74$ & 1,0 & $-0,08$ & $-0,02$ \\
\hline & & $<0,01$ & 0,03 & 0,01 & $<0,01$ & & 0,63 & 0,89 \\
\hline \multirow[t]{6}{*}{$012-206$} & SEV & $-\ldots-0,00$ & $-0,54$ & $-0,05$ & 1,00 & $-0,63$ & $-0,06$ & 0,31 \\
\hline & & --- & $\leq \mathbf{0 , 0 1}$ & $-0,76$ & & $\leq \mathbf{0 , 0 1}$ & $-0,68$ & 0,05 \\
\hline & REC & 0,18 & - & $-0,33$ & $-\underline{-0,63}$ & $-1,00$ & $-0,07$ & $-\underline{-0}, \underline{48}$ \\
\hline & & 0,26 & $\leq \mathbf{0 , 0 1}$ & $-0,03$ & $\leq \mathbf{0}, 01$ & & $-0,65$ & $<\mathbf{0}, 01$ \\
\hline & AВСPPIrel & $-0,25$ & 0,43 & $-0,27$ & $-0,06$ & $-0,07$ & $-1,00$ & $-0,02$ \\
\hline & & 0,11 & $<\mathbf{0 , 0 1}$ & 0,08 & 0,68 & 0,65 & & 0,91 \\
\hline \multirow[t]{4}{*}{ 012-207 } & SEV & $-0,34$ & $-0,24$ & $-0,43$ & 1,00 & $-\mathbf{- 0 , 8 2}$ & $-0,17$ & $-0,28$ \\
\hline & & 0,16 & 0,31 & $-0,07$ & & $\leq \mathbf{0 , 0 1}$. & $-\underline{0,50}$ & 0,26 \\
\hline & REC & 0,37 & $-0,16$ & $-0,46$ & $--0,82$ & $--1,00$ & $-0,50$ & 0,16 \\
\hline & & 0,12 & $0, \overline{52}$ & 0,05 & $<\mathbf{0 , 0 1}$ & & $\overline{0,03}$ & $0, \overline{50}$ \\
\hline
\end{tabular}

$\mathrm{NH}=$ Número de hojas después de la sequía, $\mathrm{CRA}=$ Contenido Relativo de Agua, $\mathrm{CC}=$ Contenido de clorofila presente en una hoja después de la sequía, $\mathrm{SEV}=$ Severidad tras la sequía, $\mathrm{REC}=$ Grado de recuperación tras la sequía, ABCPPIrel.=Área Bajo la Curva de Progreso de Phytophthora infestans relativa; PT=Peso de tubérculos. Las correlaciones en negrillas son aquellas altamente significativas 
Tabla 3. 54 genotipos seleccionados por resistencia a tizón y sequía, caracteres asociados a resistencia a sequía y rendimiento.

\begin{tabular}{|c|c|c|c|c|c|c|c|c|}
\hline Genotipo & NH & CRA & $\mathrm{CC}$ & SEV & REC & ABCPPIrel. & NTU & PT \\
\hline $012-200-7$ & 7 & 52,94 & 32,3 & 3 & 5 & 0,75 & 5 & 0,04 \\
\hline 012-200-16 & 9 & 42,86 & 44,5 & 2 & 5 & 14,85 & 7 & 0,05 \\
\hline $012-200-24$ & 7 & 57,89 & 27,6 & 4 & 3 & 16,95 & 19 & 0,06 \\
\hline 012-200-25 & 5 & 50 & 40 & 3 & 4 & 17,6 & 16 & 0,05 \\
\hline $012-201-2$ & 6 & 43,48 & 41,5 & 4 & 4 & 20,15 & 4 & 0,04 \\
\hline 012-201-4 & 9 & 52,38 & 44,2 & 3 & 4 & 27,05 & 9 & 0,09 \\
\hline 012-201-9 & 10 & 50 & 44,3 & 4 & 3 & 13,95 & 4 & 0,1 \\
\hline 012-201-11 & 6 & 40,91 & 26,7 & 4 & 1 & 10,6 & 8 & 0,09 \\
\hline 012-201-12 & 7 & 44,12 & 40,4 & 3 & 3 & 12,15 & 6 & 0,08 \\
\hline 012-201-13 & 10 & 67,74 & 43,8 & 2 & 5 & 5,65 & 4 & 0,15 \\
\hline 012-201-14 & 12 & 57,69 & 43 & 3 & 5 & 27,7 & 8 & 0,06 \\
\hline 012-201-16 & 11 & 55,56 & 47,6 & 3 & 4 & 14,8 & 19 & 0,1 \\
\hline 012-201-17 & 13 & 50 & 43,2 & 3 & 4 & 18,5 & 7 & 0,09 \\
\hline 012-201-21 & 7 & 50 & 34,2 & 4 & 3 & 0,6 & 17 & 0,05 \\
\hline 012-201-23 & 9 & 37,5 & 31,1 & 3 & 3 & 14,75 & 11 & 0,09 \\
\hline $012-201-28$ & 4 & 63,64 & 34 & 3 & 4 & 3,15 & 14 & 0,05 \\
\hline $012-201-38$ & 8 & 50 & 39,9 & 3 & 4 & 15,2 & 5 & 0,11 \\
\hline $012-201-43$ & 5 & 72,22 & 38,7 & 2 & 3 & 20,75 & 7 & 0,1 \\
\hline 012-201-44 & 3 & 56,1 & 37,1 & 2 & 5 & 16 & 7 & 0,07 \\
\hline 012-201-45 & 13 & 71,43 & 45,3 & 2 & 5 & 7,8 & 6 & 0,04 \\
\hline 012-201-50 & 14 & 59,26 & 49,2 & 2 & 5 & 17,5 & 12 & 0,09 \\
\hline 012-201-51 & 9 & 60,87 & 33,4 & 3 & 2 & 11,55 & 12 & 0,07 \\
\hline 012-201-52 & 12 & 50 & 48,5 & 4 & 3 & 13,15 & 16 & 0,08 \\
\hline 012-201-53 & 10 & 50 & 43,7 & 2 & 5 & 22,55 & 11 & 0,06 \\
\hline $012-201-54$ & 2 & 71,43 & 44 & 2 & 5 & 17,5 & 15 & 0,06 \\
\hline 012-201-55 & 12 & 60,71 & 44,8 & 3 & 4 & 18 & 17 & 0,08 \\
\hline 012-201-56 & 7 & 61,29 & 47,5 & 3 & 4 & 9,8 & 8 & 0,05 \\
\hline 012-201-57 & 11 & 55,56 & 47,3 & 3 & 4 & 10,95 & 7 & 0,07 \\
\hline 012-202-1 & 17 & 77,78 & 39,8 & 2 & 5 & 17,3 & 4 & 0,06 \\
\hline $012-202-6$ & 16 & 44 & 49,8 & 3 & 5 & 18,1 & 8 & 0,04 \\
\hline $012-202-7$ & 15 & 52 & 48,2 & 2 & 5 & 17,05 & 1 & 0,02 \\
\hline $012-202-8$ & 5 & 69,23 & 39,8 & 2 & 4 & 13,1 & 2 & 0,02 \\
\hline 012-202-9 & 15 & 66,67 & 37,4 & 3 & 4 & 18,4 & 6 & 0,04 \\
\hline 012-202-11 & 7 & 60 & 43,4 & 3 & 4 & 24,8 & 9 & 0,07 \\
\hline 012-202-15 & 3 & 65,22 & 46,8 & 3 & 5 & 20,3 & 8 & 0,06 \\
\hline 012-202-16 & 14 & 57,89 & 44,3 & 3 & 5 & 18,5 & 10 & 0,06 \\
\hline 012-202-17 & 18 & 50 & 48,9 & 3 & 3 & 23,85 & 15 & 0,06 \\
\hline 012-202-18 & 12 & 51,85 & 40,5 & 3 & 4 & 11,15 & 10 & 0,06 \\
\hline 012-202-20 & 17 & 62,86 & 45,8 & 3 & 5 & 22,75 & 15 & 0,08 \\
\hline 012-202-21 & 14 & 54,55 & 43,5 & 3 & 4 & 11,1 & 4 & 0,05 \\
\hline 012-202-22 & 19 & 54,55 & 35,1 & 3 & 5 & 11,35 & 2 & 0,03 \\
\hline $012-202-23$ & 15 & 61,11 & 43 & 3 & 4 & 16,7 & 5 & 0,04 \\
\hline 012-202-24 & 14 & 64,52 & 35,2 & 3 & 4 & 20,25 & 6 & 0,02 \\
\hline 012-202-25 & 11 & 60 & 42,8 & 2 & 4 & 11,7 & 0 & 0 \\
\hline 012-202-26 & 12 & 57,14 & 42,2 & 3 & 5 & 25,1 & 12 & 0,07 \\
\hline 012-202-27 & 16 & 50 & 40,6 & 2 & 4 & 8,6 & 11 & 0,04 \\
\hline 012-202-29 & 17 & 62,96 & 41,4 & 2 & 5 & 17,5 & 3 & 0,05 \\
\hline $012-202-31$ & 14 & 60 & 41,2 & 3 & 5 & 19,6 & 7 & 0,04 \\
\hline $012-202-33$ & 13 & 61,29 & 37,3 & 3 & 4 & 20,2 & 5 & 0,07 \\
\hline $012-202-34$ & 9 & 55,17 & 41,1 & 2 & 5 & 18,65 & 7 & 0,07 \\
\hline 012-202-35 & 16 & 62,07 & 48 & 3 & 5 & 12,4 & 5 & 0,09 \\
\hline 012-202-36 & 10 & 45,83 & 37,8 & 2 & 5 & 16,45 & 7 & 0,04 \\
\hline $012-205-49$ & 6 & 52,38 & 25,7 & 3 & 3 & 8,05 & 6 & 0,05 \\
\hline $012-205-52$ & 8 & 52,17 & 33,5 & 3 & 4 & 15,95 & 8 & 0,07 \\
\hline
\end{tabular}


Tabla 4. Aptitud Combinatoria General de dos progenitores masculinos de papa para seis variables de resistencia a tizón y sequía.

\begin{tabular}{|c|c|c|c|c|c|c|}
\hline \multicolumn{7}{|c|}{ Aptitud Combinatoria General } \\
\hline Progenitor & $\mathrm{NH}^{* *}$ & $\mathrm{CRA}^{* *}$ & $\mathrm{CC} * *$ & $\mathrm{SEV}^{* *}$ & REC & ABCPPIrel** \\
\hline CCC81 & $1, \mathbf{1 8}^{\mathbf{a}}$ & $5,56 \mathbf{a}$ & $3,55^{\mathrm{a}}$ & $-0,20^{a}$ & $0,30 \mathrm{a}$ & $-11,40 a$ \\
\hline DB-22670 & $-1,18 b$ & $-5,56 b$ & $-3,55 b$ & $0,20 \mathrm{~b}$ & $-0,30^{\mathrm{a}}$ & $11,40 \mathrm{~b}$ \\
\hline
\end{tabular}

**=altamente significativo $(\mathrm{p}<0,01), \mathrm{NH}=$ Número de hojas después de la sequía, $\mathrm{CRA}=$ Contenido Relativo de Agua, $\mathrm{CC}=$ Contenido de clorofila presente en una hoja después de la sequía, SEV=Severidad tras la sequía, REC=Potencial de recuperación tras la sequía, ABCPPIrel=Área Bajo la Curva de Progreso de Phytophthora infestans relativa.

Tabla 5. Heredabilidades y bases genéticas para seis progenies de cruzas inter específicas de papa evaluadas para seis variables de resistencia a sequía y tizón.

\begin{tabular}{cccccccc}
\hline & \multicolumn{7}{c}{ Heredabilidades } \\
\hline & $\mathrm{NH}$ & CRA & CC & SEV & REC & ABCPPIrel. \\
\hline $\mathrm{H}^{2}$ & & 0,75 & 0,46 & 0,66 & 0,38 & 0,40 & 0,71 \\
$\mathrm{~h}^{2}$ & & 0,07 & 0,46 & 0,66 & 0,23 & 0,10 & 0,71 \\
\hline Importancia de los & A/NA & 0,10 & infinito & Infinito & 1,62 & 0,32 & Infinito \\
efectos genéticos & NA/A & 9,98 & 0 & 0 & 0,62 & 3,12 & 0 \\
\hline$H^{2}$
\end{tabular}

$\mathrm{H}^{2}=$ Heredabilidad en sentido amplio, $\mathrm{h}^{2}=$ Heredabilidad en sentido estrecho, $\mathrm{NA}=$ Efectos no aditivos, $\mathrm{A}=$ Efectos aditivos, NH=Número de hojas después de la sequía, CRA=Contenido Relativo de Agua, $\mathrm{CC}=\mathrm{Contenido}$ de clorofila presente en una hoja después de la sequía, SEV=Severidad tras la sequía, REC=Potencial de recuperación tras la sequía, ABCPPIrel.=Área Bajo la Curva de Progreso de Phytophthora infestans relativa. 


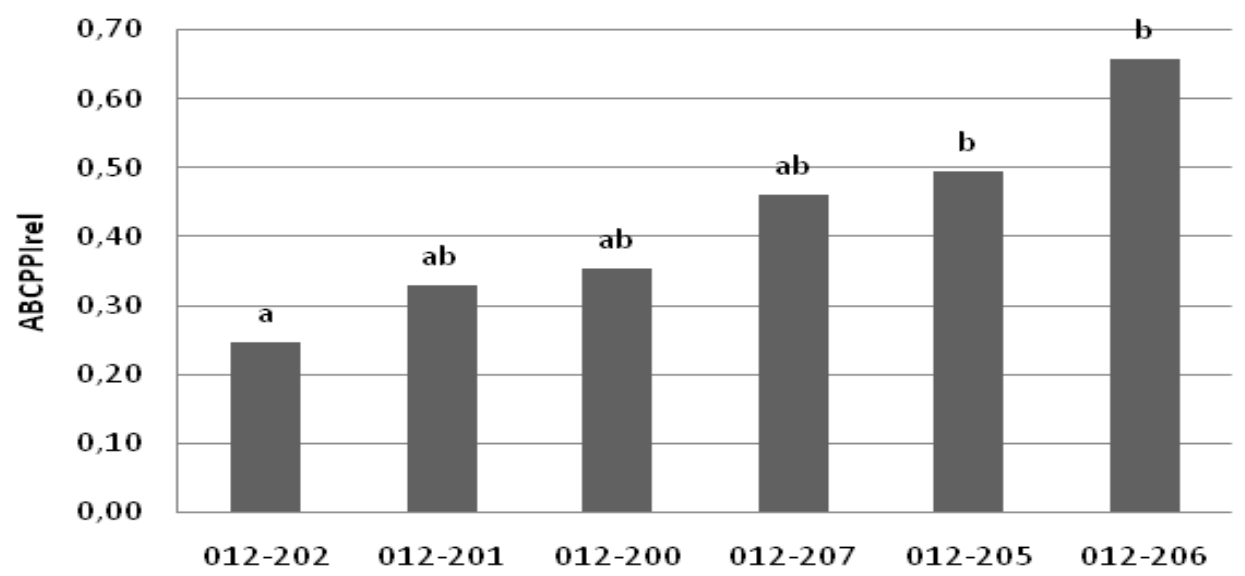

Figura 1.Área bajo la curva de progreso de Phytophtora infestans (ABCPPIrel) para seis familias de cruzas inter-específicas de papa evaluadas para resistencia a tizón. Las medias con la misma letra son estadísticamente iguales $(\mathrm{p}<0,01)$.

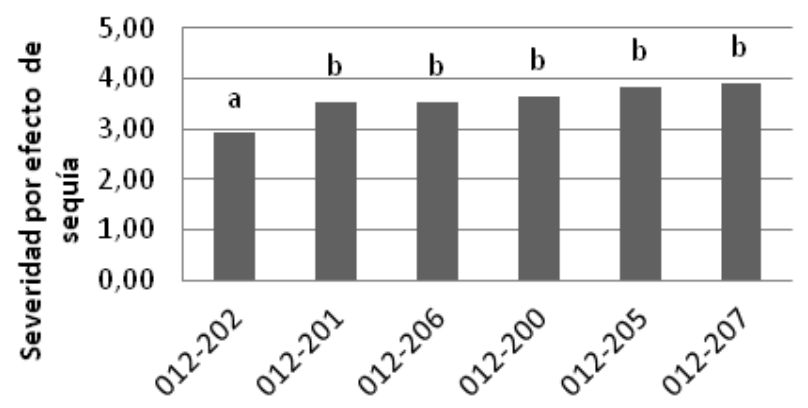

Figura 2. Grado de severidad para seis familias de cruzas inter-específicas de papa sometidas a sequía

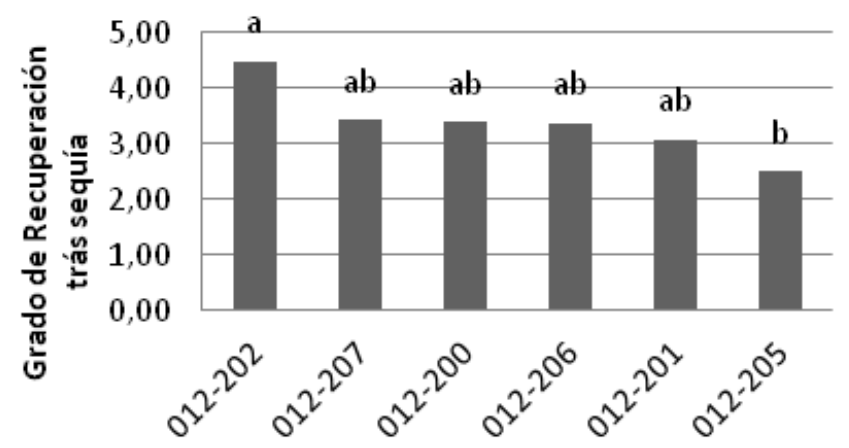

Figura 3. Grado de recuperación para seis familias de cruzas interespecífica de papa sometidas a sequía. 
13 Vol 20 (2) 2016 Resistencia genética en familias de híbridos de papa a tizón y sequía

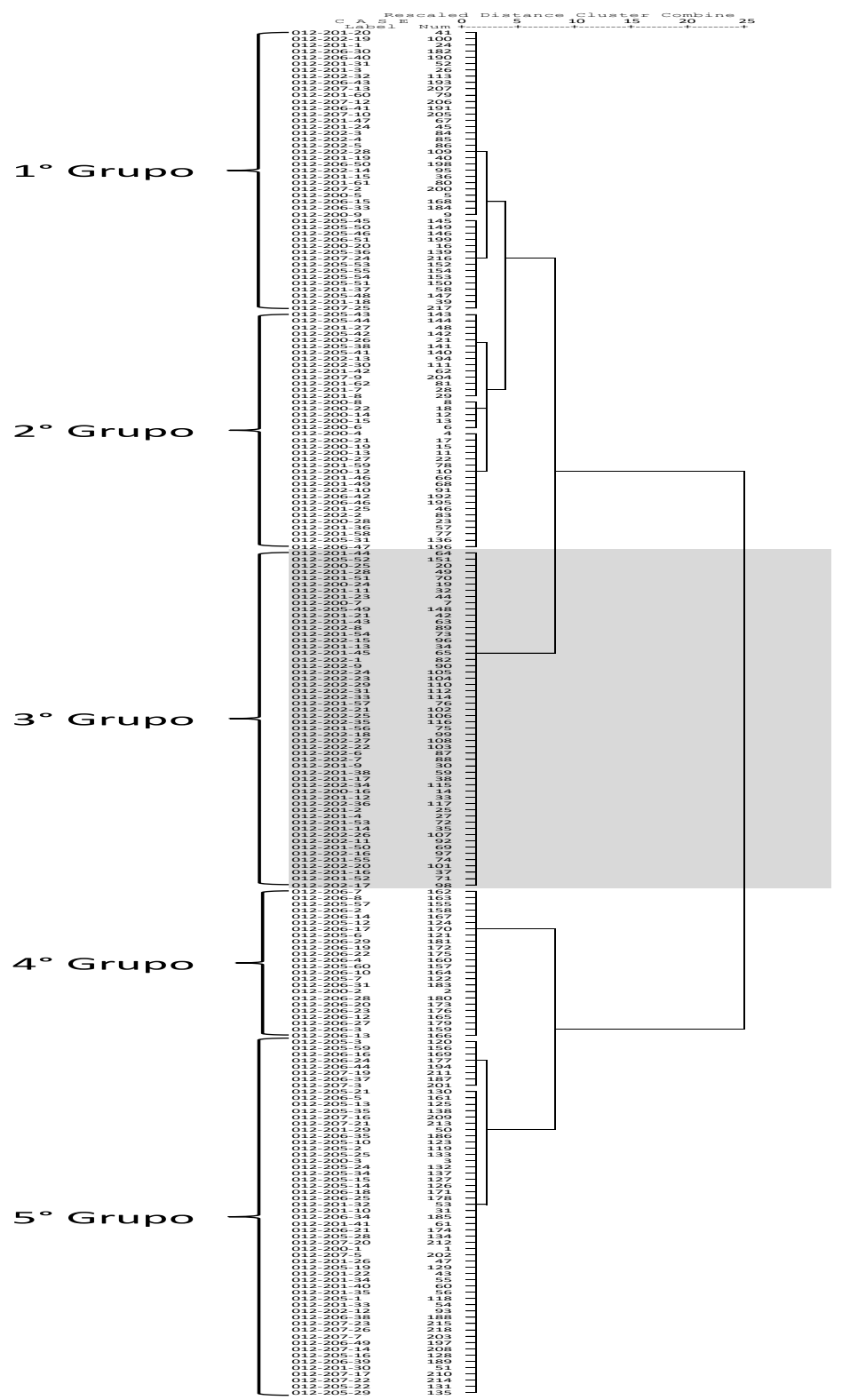

Figura 4. Dendograma de agrupamiento jerárquico para clones. 